\title{
Fingerprint Recognition System Based on Mapping Approach
}

\author{
Dayashankar Singh \\ M.M.M. Engineering College \\ Gorakhpur (UP)
}

\author{
Dr. P.K.Singh \\ M.M.M. Engineering College \\ Gorakhpur (UP)
}

\author{
Dr. R.K.Shukla \\ M.M.M. Engineering College \\ Gorakhpur (UP)
}

\begin{abstract}
Fingerprint identification system is mainly consisted of fingerprint achieving, fingerprint classification and fingerprint matching. Fingerprint matching is the key to the system and effects on the precision and efficiency of the whole system directly. Fingerprints are matched mainly based on their fingerprint texture pattern which can be described with the orientation field of fingerprints. A fingerprint, which has the different orientation angle structure in different local area of the fingerprint and has a texture pattern correlation among the neighboring local areas of the fingerprint, can be viewed as a Markov stochastic field. A novel method of fingerprint matching, which is based on embedded Hidden Markov Model (HMM) that is used for modeling the fingerprint's orientation field, is described in this paper. The accurate and robust fingerprint matching can be achieved by matching embedded Hidden Markov Model parameters which were built after the processing of extracting features from a fingerprint, forming the samples of observation vectors and training the embedded Hidden Markov Model parameters.
\end{abstract}

\section{Keywords}

Fingerprint identification, Fingerprint matching, Hidden Markov Model, Orientation field.

\section{INTRODUCTION}

In this paper, Fingerprint time attendance system has been developed which is based on fingerprint identification systems and effects on the precision and efficiency of the whole systems directly. For all systems, the following issue is of great interest: how to extract and utilize robust features of fingerprints. According to the different types of features, previous approaches to fingerprint matching can be roughly divided into two categories: (1) the conventional approach based on minutiae of fingerprints [1], and (2) the statistical approach based on fingerprint texture structure [2]. The approaches based on minutiae of fingerprints, which includes ends and bifurcations of fingerprint ridges, represent fingerprints with the properties and relative positions of minutiae of a fingerprint. These algorithms above are reliably when the input fingerprint images are of good qualities. When the quality of fingerprint images is poor, the performance of these algorithms degrades rapidly, because there have many pseudo minutiae and unreliable ridge features. A fingerprint is different from the other fingerprints in that it possesses unique texture structure, which can be described with the orientation field of fingerprints. A fingerprint has the different orientation angle structure in different local area of the fingerprint and has a texture pattern correlation among the neighboring local areas of the fingerprint. It is true that different samples selected from the same fingerprint are very similar and obey a statistical probability distribution, though they aren't as like as two peas due to the existence of distortion and noise. The fingerprint matching based on statistical approaches is usually insensitive to the quality of fingerprint images. A fingerprint texture with specified details changing across a predefined path on the finger surface can be viewed as a Markov chain. Hidden Markov Model (HMM) is expected to be an efficient approach in fingerprint matching, since it has been successfully applied to speech recognition and other recognition systems [3], [4]. Andrew Senior presented a HMM approach in fingerprint classification, and verified that the HMM approach is effective [5], [6]. Andrew Senior's approach uses a number of fingerprint parameters; some of them are related to ridge details, which will be extracted only after smoothing and thinning processes and that is time consuming. Here we are going to improving the accuracy of the Fingerprint time attendance system using additional phase i.e. Mapping. An orientation of describing the print texture pattern, which can be expressed as a discrete orientation angle series along a predefined path region chain. In this paper a new Mapping based HMM based fingerprint matching approach using only orientation angle parameters is presented. An Experiment has been carried out by collecting various samples from various people. Experiment shows that the developed technique gives better recognition accuracy. It also minimizes the False Acceptance Ratio (FAR). It has also been observed that this technique requires very less time to recognize the fingerprints of a person. Hidden Markov Model (HMM) may be used for training samples (Train Data) and testing the samples (Test Data) during the development of Fingerprint recognition system.

\section{THE EMBEDDED HMM FOR A FINGERPRINT IMAGE}

A HMM provides a statistic model for a set of observation data sequences [5]. It includes two forms of stochastic finite process. One is a Markov chain of finite state, which describes the transfer from one state to another; the other describes the probabilities between states and observation data. What is necessary to statistically characterize a HMM is a state transition probability matrix, an initial state probability distribution, and a set of probability density functions associated with the observations for each state. Typically a HMM is a 1-D 
structure suitable for analyzing 1-D random signals, for example speech signals.

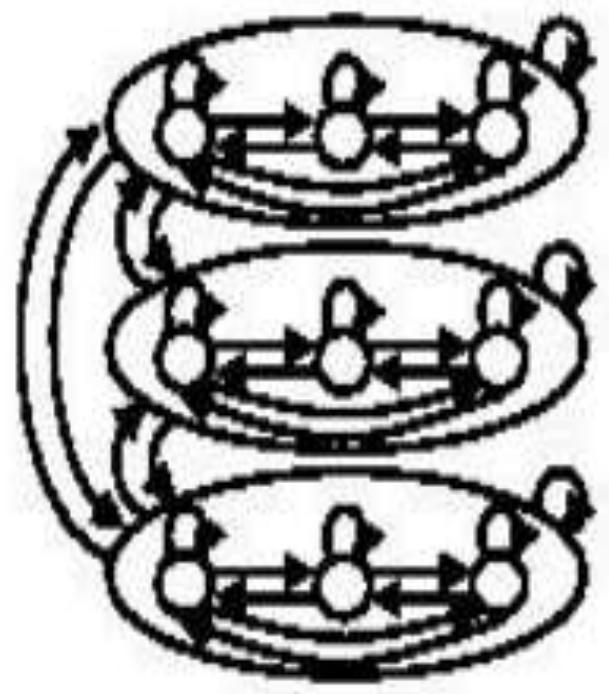

Figure 1.Topology structure of an embedded HMM

A 1-D HMM can be developed to a pseudo 2-D structure (Figure 1) by extending each state in a 1-D HMM as a sub HMM. In this way the HMM consists of a set of super states, along with a set of embedded states. This pseudo 2-D HMM is also called embedded HMM. The super states were used to model 2-D data along one direction, with the embedded HMM modeling the data along the other direction.

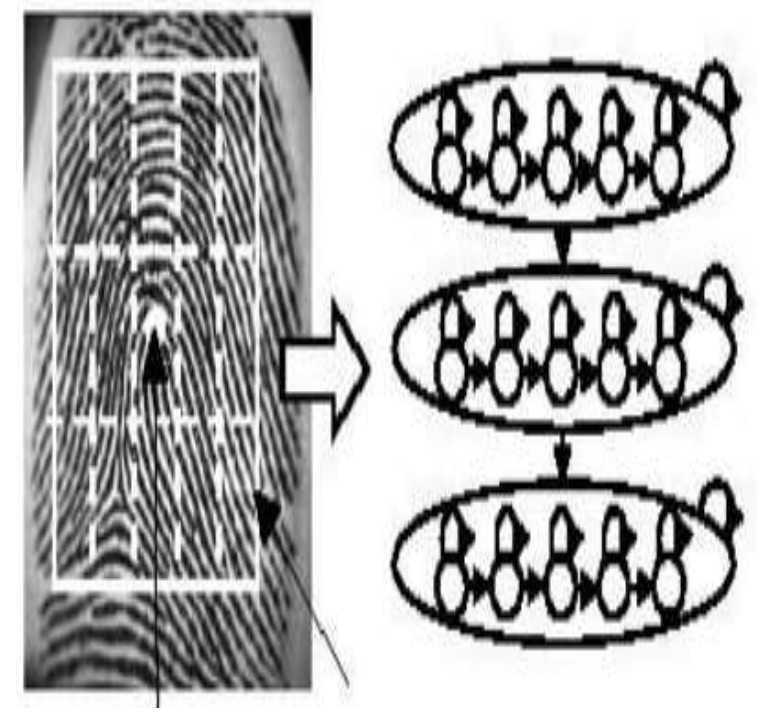

Reference point Reference area of a fingerprint

Figure 2.The embedded HMM of the fingerprint

In this paper the embedded HMM scheme of an input Fingerprint image is shown as Figure 2. The embedded HMM includes three super states, which represent three parts of a finger print from the top to bottom. Each super state is composed of five sub states (embedded states) horizontally.

\section{FEATURE EXTRACTION AND OBSERVATION VECTOR FORMATION}

Fingerprint image discretization by sampling
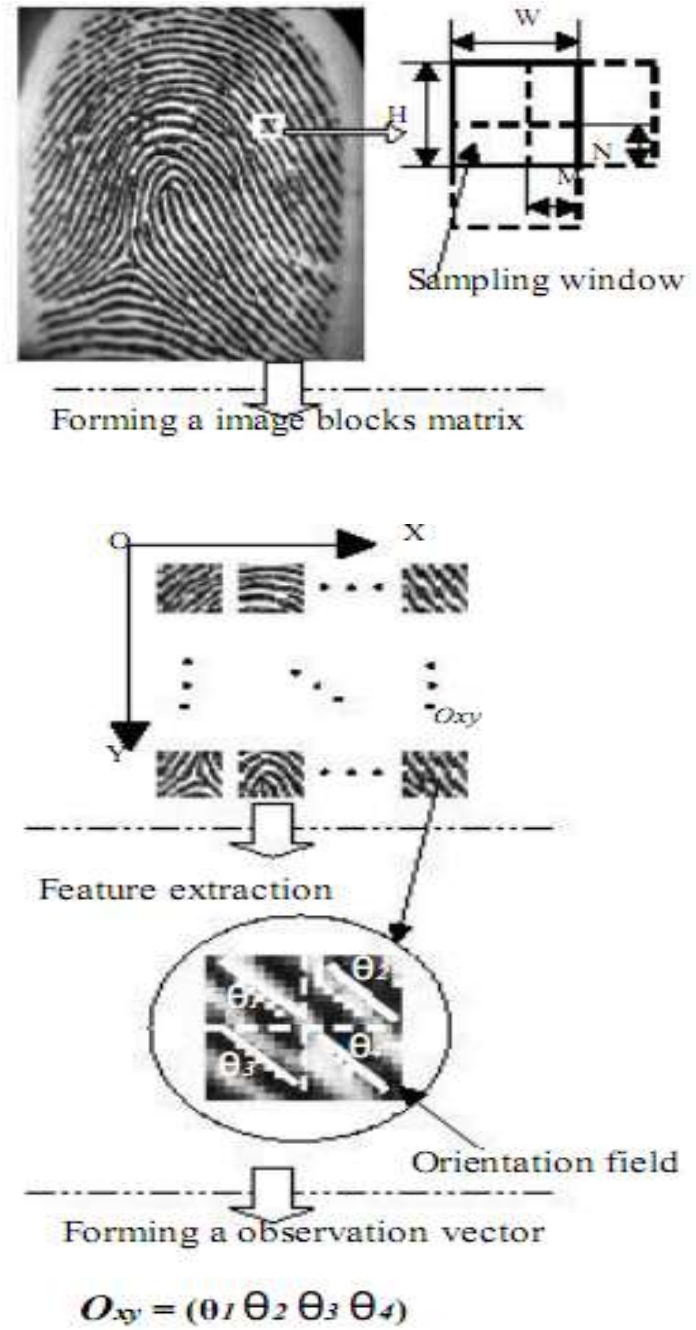

Figure 3.Feature extraction and observation vector formation

The detail of the feature extraction and HMM observation vector formation has been described in reference [7]. Generally speaking, it includes the following steps (Figure 3):

\section{Step I: Fingerprint image discretization}

A fingerprint image is scanned with a $\mathrm{W} \times \mathrm{H}$ sampling window (image block) left to right and top to bottom. The overlap between adjacent windows is $\mathrm{N}$ pixels distance in the vertical direction and $\mathrm{M}$ pixels distance in the horizontal direction. In 
this way a fingerprint can be divided into a $\mathrm{Y} \times \mathrm{X}$ image blocks matrix, and the size of each block is $\mathrm{W} \times \mathrm{H}$. This technique can improve the ability of an embedded HMM to model the neighborhood relations between the sampling windows.

\section{Step II: Constructing the observation vectors}

Each W x H image block includes four sub image blocks. By estimating the local orientation of each sub block, we can get an observation vector ( $\left.\begin{array}{llll}1 & 2 & 3 & 4\end{array}\right)$, which can described the features of the $\mathrm{W} \times \mathrm{H}$ image block reasonably.

\section{StepIII: Forming the observation vector sequence}

Each W x H image block can be represented with an observation vector ( $\left.\begin{array}{llll}1 & 2 & 3 & 4\end{array}\right)$, and a fingerprint image can be divided into an $\mathrm{Y} \times \mathrm{X}$ image blocks matrix, so a fingerprint can be described with an 4 x Y x X observation vector sequence.

\section{PRETREATMENT}

Since a captured fingerprint appears randomly in an image, a pretreatment is needed for adjusting the location of a fingerprint and setting the reference area, which includes the whole area for fingerprint feature extraction. The basic steps of a pretreatment are (Figure 4) [8]:

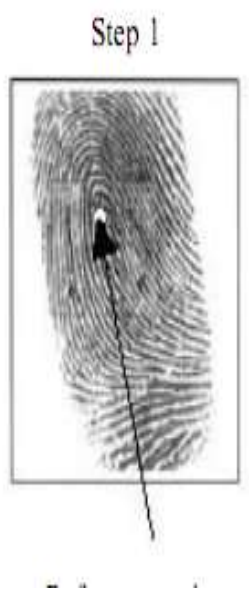

Reference point

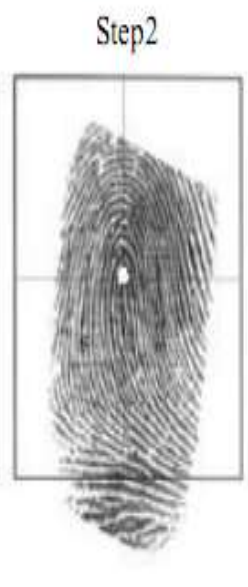

Reference area
Figure 4. The pretreatment of the location of a fingerprint

\section{Step I:}

A reference point of a fingerprint is detected, and the location of the reference point is adjusted to the image center. The reference point detection algorithm based on the orientation field of fingerprint images has been described in reference [9] and is not detailed here.

\section{Step II:}

The fingerprint is rotated based on the ridges structure around the reference point, which has been described in the M82 [10].

\section{Step III:}

After the location of a fingerprint is adjusted, a reference area (Figure 2, 4) can be set.

\section{HMM TRAINING AND FINGERPRINT MATCHING}

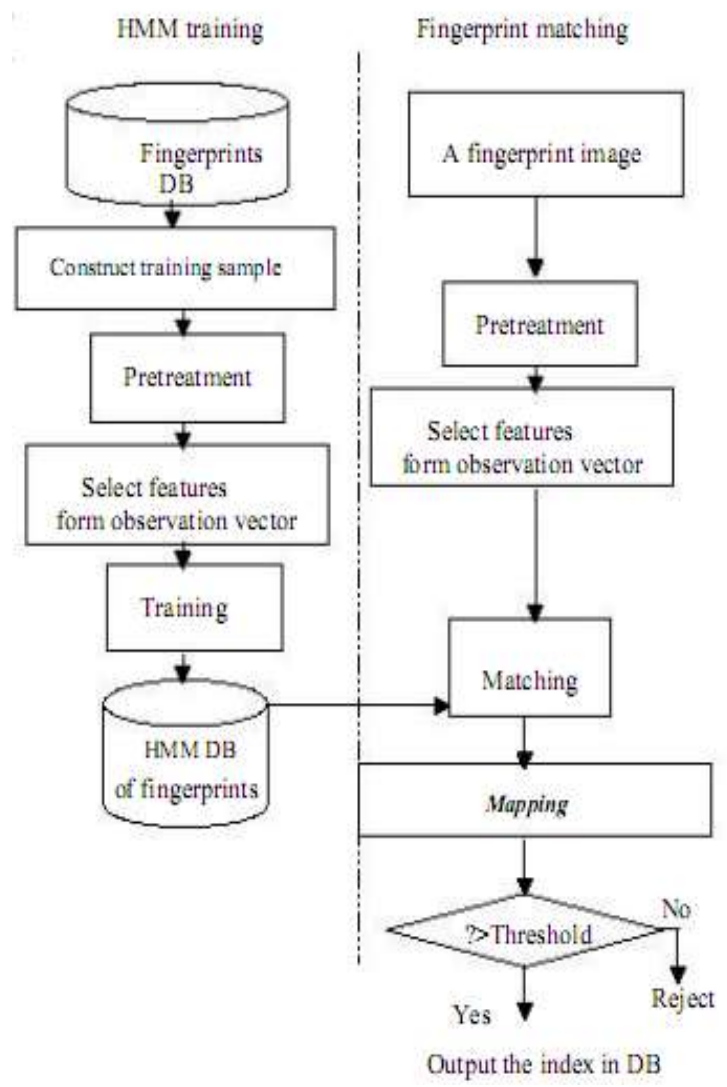

Figure 5. Training and matching for fingerprints based on HMM

Samples from the same fingerprint correspond to an embedded HMM and different fingerprints correspond to different embedded HMM in HMM DB of fingerprints. The embedded HMM is built by selecting different samples of the same fingerprints and implementing a training processing, which is a process for estimating the corresponding embedded HMM parameters. Fingerprint matching processing of a test fingerprint is to find the maximum matching likelihood of the embedded HMM among different fingerprints. The general training and matching process scheme is summarized shown in Figure 5. The main procedures in training and matching process are re-segmentation and iterative re-estimations of model parameters. Traditional HMM technique solves those problems with Viterbi algorithm and Baum-Welch algorithm [3]. 


\section{SETTING THE THRESHOLD OF REJECTION}

In matching processing a fingerprint for matching is input, and the maximum matching likelihood of the embedded HMM among different fingerprints is found in the HMM DB of fingerprints. While the maximum matching likelihood just implies that the fingerprint for matching resembles a template in fingerprint $\mathrm{DB}$ to a degree, and cannot decide ultimately that the fingerprint and a template are all from the same fingerprint.

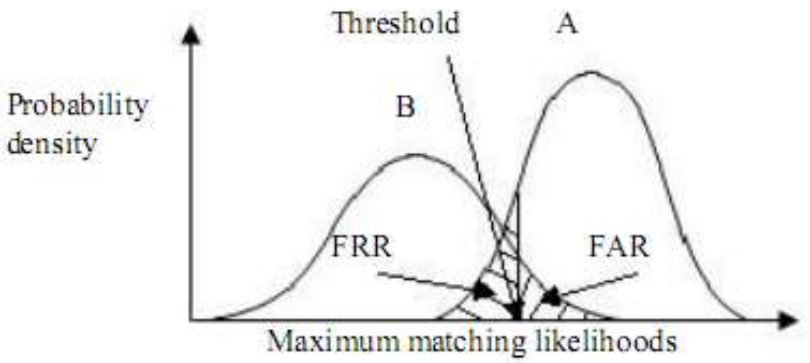

Figure 6. Distribution of maximum matching likelihoods

As for the different samples of the same fingerprint, their maximum matching likelihoods should obey a probability distribution. For example, the mark " $A$ " and "B" denote the two different fingerprints likelihoods distribution in Figure 6, the maximum matching likelihoods of Fingerprint A and B obey the normal distribution. There is a overlap between the two probability distribution curves, which means a fingerprint sample for matching likely belong to A or B in the overlap area. In order to decide the identity of the fingerprint sample, the threshold of rejection should be set. When the maximum matching likelihood the sample is up the threshold, the identity of the fingerprint sample can be decided. Usually, the confidence coefficient of a probability distribution is used as the threshold. The False Acceptance Rate (FAR) and False Rejection Rate (FRR) are also changed accordingly when the threshold is changed. A threshold of rejection is set when the confidence coefficient of a normal distribution is $99 \%$. Table 1 six Logarithm values of maximum matching likelihoods correspond to six fingerprint training samples of a fingerprint.

Table1. Setting the threshold of rejection

\begin{tabular}{l|l|l|c}
\hline $\begin{array}{c}\text { Logarithm of maximum } \\
\text { Matching }\end{array}$ & $\begin{array}{l}\text { Mean } \\
\text { Value }\end{array}$ & $\begin{array}{c}\text { Mean } \\
\text { square (V) }\end{array}$ & $\begin{array}{c}\text { Threshold of } \\
\text { rejection }\end{array}$ \\
\hline$-9962.1,-9904.4$ & -9938.3 & 4436.1 & -1003.0 \\
$-10001.4,-9877.1$ & & & \\
$-10022.1,-9862.7$ & & & \\
\hline
\end{tabular}

\section{EXPERIMENT RESULTS}

A fingerprint matching system based on the above algorithm has been developed and tested on FVC2002-DB1, which includes 880 ( $110 \times 8$, which means 110 different fingerprints and 8 samples per fingerprint) fingerprint images with 374 x 388 pixels in 256 gray scale levels. Six samples per fingerprint were selected randomly as a training set, and the other two samples were for testing set. Training set was used for training and estimating the corresponding embedded HMM parameters. In this way 110 HMM were formed. A total of $24200(110 \times 220)$ matching were performed. Table 2 shows the value of FAR and FRR under the different confidence coefficient. The ROC is shown in Figure 7, and the Equal Error Rate (EER) is about 7.1\%.

Table 2. The value of FAR and FRR

\begin{tabular}{c|c|c}
\hline Confidence coeff. & FAR & FRR \\
\hline $99.0 \%$ & 0 & $23.85 \%$ \\
$99.5 \%$ & $4.18 \%$ & $9.93 \%$ \\
$99.9 \%$ & $8.91 \%$ & $5.19 \%$ \\
\hline
\end{tabular}

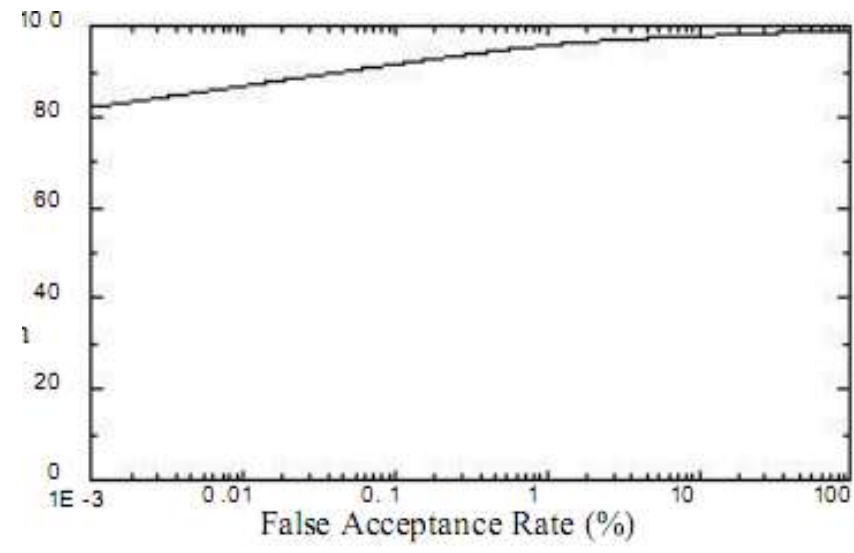

Figure 7: False Acceptance Ratio

\section{CONCLUSIONS}

Fingerprint matching is the key to fingerprint identification systems and effects on the precision and efficiency of the whole system directly. The paper presents a fingerprint matching approach based on the embedded HMM approach, the system has the following characteristics:

- The performance is good and robust. The matching only depends on the orientation field of a fingerprint, so it is less sensitive to the noise and distortions of a fingerprint 
image than the conventional approaches in which the dependent parameters include more fingerprint details.

- The pretreatment process is simple. The algorithm skipped the processes of thinning the ridge image and selecting minutiae. The enhancement of the fingerprint is also not necessary usually.

\section{REFERENCES}

[1] N. Ratha, K. Karu, S. Chen, A. K. Jain, "A real-time matching system for large fingerprint databases", IEEE Transactions on Pattern Analysis and Machine Intelligence, Vol. 18, No. 8, pp. 799-813, 1996.

[2] Salil Prabhakar, "Fingerprint classification and matching using a filterbank", Michigan State University, Michigan, USA, 2001.

[3] Andrew Senior, "A Combination Fingerprint Classifier" IEEE Transactions on pattern analysis and machine intelligence Vol. 10, No. 23, pp. 1165-1174, 2001.

[4] Andrew Senior, "A Hidden Markov Model Fingerprint Classifier", In proceedings of the 31st Asilomar conference on Signals, Systems and Computers, pp. 306-310, 1997.
[5] Nefian A V, HayesIII M H, "Face recognition using an Embedded-HMM", Proceedings of IEEE Internationa Conference on Audio and Video-based Biometric Person Authentication, Washington D.C.USA, pp. 19-21, 1999.

[6] Rabiner L, "A tutorial on HMM and selected applications in speech recognition", Proc. IEEE, Vol. 77, No. 2, pp. 257-286 1989.

[7] Guo Hao , Ou ZongYing, He Yang, "Automatic fingerprint classification based on embedded Hidden Markov Models" International Conference on Machine Learning and Cybernetics Vol. 5, pp. 3033-3038, 2003.

[8] Ou Zongying, Guo Hao, Wei Honglei, "Fingerprint classifie using embedded Hidden Markov Models", Sinobiometrics " 04, pp 517-522, 2004.

[9] Chul-Hyun Park, Sang-Keun Oh, Dong-Min Kwak, Bum-Soo Kim, Young-Chul Song, Kil-Houm Park, "A New Referenc Point Detection Algorithm Based on Orientation Pattern Labeling in Fingerprint Images", IBPRIA, pp. 697-703, 2003.

[10] D. Maltoni, D. Maio, Anil K. Jain, Salil Prabhakar, "HandbookofFingerprintRecognition", Springer-Verlag, New York, USA, 2003. 